Ferrata Storti Foundation

\title{
Red blood cell metabolism in Rhesus macaques and humans: comparative biology of blood storage
}

\author{
Davide Stefanoni, ${ }^{1}$ Hye Kyung H. Shin, ${ }^{2}$ Jin Hyen Baek, ${ }^{2}$ Devin P. Champagne, ${ }^{1}$ \\ Travis Nemkov, ${ }^{1}$ Tiffany Thomas, ${ }^{3}$ Richard O. Francis, ${ }^{3}$ James C. Zimring, ${ }^{4}$ \\ Tatsuro Yoshida, ${ }^{5}$ Julie A. Reisz, ${ }^{1}$ Steven L. Spitalnik, ${ }^{3}$ Paul W. Buehler ${ }^{2}$ and \\ Angelo D'Alessandro ${ }^{1,6}$ \\ ${ }^{1}$ Department of Biochemistry and Molecular Genetics, University of Colorado Denver - \\ Anschutz Medical Campus, Aurora, CO; ${ }^{2}$ Center for Biologics Evaluation and Research, \\ Food and Drug Administration, Silver Spring, MD; ${ }^{3}$ Department of Pathology \& Cell Biology,

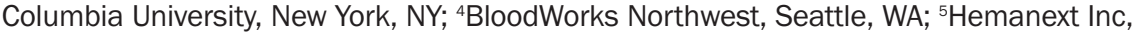 \\ Lexington, MA and ${ }^{6}$ Department of Medicine, Division of Hematology, University of \\ Colorado Denver - Anschutz Medical Campus, Aurora, CO, USA
}

Haematologica 2020

Volume 105(8):2174-2186

\section{Correspondence:}

ANGELO D'ALESSANDRO

angelo.dalessandro@ucdenver.edu

PAUL W. BUEHLER,

Paul.Buehler@fda.hhs.gov

Received: June 19, 2019.

Accepted: October 10, 2019.

Pre-published: November 7, 2019.

doi:10.3324/haematol.2019.229930

Check the online version for the most updated information on this article, online supplements, and information on authorship \& disclosures: www. haematologica.org/content/105/8/2174

(C)2020 Ferrata Storti Foundation

Material published in Haematologica is covered by copyright. All rights are reserved to the Ferrata Storti Foundation. Use of published material is allowed under the following terms and conditions:

https://creativecommons.org/licenses/by-nc/4.0/legalcode. Copies of published material are allowed for personal or internal use. Sharing published material for non-commercial purposes is subject to the following conditions:

https://creativecommons.org/licenses/by-nc/4.0/legalcode, sect. 3. Reproducing and sharing published material for commercial purposes is not allowed without permission in writing from the publisher.

\section{ABSTRACT}

M acaques are emerging as a critical animal model in transfusion medicine, because of their evolutionary similarity to humans and perceived utility in discovery and translational science. However, little is known about the metabolism of Rhesus macaque red blood cells (RBC) and how this compares to human RBC metabolism under standard blood banking conditions. Metabolomic and lipidomic analyses, and tracing experiments with $\left[1,2,3-{ }^{13} \mathrm{C}_{3}\right]$ glucose, were performed using fresh and stored RBC (sampled weekly until storage day 42) obtained from Rhesus macaques $(n=20)$ and healthy human volunteers $(n=21)$. These results were further validated with targeted quantification against stable isotope-labeled internal standards. Metabolomic analyses demonstrated inter-species differences in RBC metabolism independent of refrigerated storage. Although similar trends were observed throughout storage for several metabolic pathways, species- and sex-specific differences were also observed. The most notable differences were in glutathione and sulfur metabolites, purine and lipid oxidation metabolites, acylcarnitines, fatty acyl composition of several classes of lipids (including phosphatidylserines), glyoxylate pathway intermediates, and arginine and carboxylic acid metabolites. Species-specific dietary and environmental compounds were also detected. Overall, the results suggest an increased basal and refrigerator-storage-induced propensity for oxidant stress and lipid remodeling in Rhesus macaque RBC cells, as compared to human red cells. The overlap between Rhesus macaque and human RBC metabolic phenotypes suggests the potential utility of a translational model for simple RBC transfusions, although inter-species storage-dependent differences need to be considered when modeling complex disease states, such as transfusion in trauma/hemorrhagic shock models.

\section{Introduction}

Rhesus macaques (Macaca mulatta, hereafter RM) are one of the most thoroughly studied non-human primates, in part because of their broad geographic distribution, reaching from Afghanistan and India, and across to China. In addition, RM evolutionarily diverged from human ancestors $\sim 25$ million years ago (by comparison, rodents and human ancestors diverged $\sim 70$ million years ago). Indeed, macaques share an average sequence identity of $\sim 93 \%$ with Homo sapiens ${ }^{1}$ and preclinical models often rely on macaques to investigate mechanisms and test interventions in the context of leading causes of human disease, including trauma/hemorrhagic shock, ${ }^{2}$ human immunodeficiency virus, ${ }^{3}$ cancer, and cardiovascular disease. ${ }^{4}$ For example, 
by metabolomic phenotyping plasma from rodents, swine, macaques, and humans, ${ }^{2}$ hemorrhage in macaques was found to recapitulate human metabolic dysfunction in trauma ${ }^{5}$ more closely than other animal species. In transfusion medicine, the study of non-human primates facilitated a landmark discovery by Landsteiner and Wiener in the late 1930s, i.e,. identification of the Rhesus blood group - after a factor found in Rhesus monkey blood. ${ }^{\circ}$

Red blood cell (RBC) transfusions can be modeled in animal species prior to pursuing costly and complex human clinical trials, or when human studies are deemed unethical (e.g., in acute radiation sickness). Nonetheless, animal modeling requires a rational approach toward species selection; in particular, blood group system diversity and variations in RBC physiology and biophysical properties across species/strains provide unique challenges when interpreting animal data in transfusion medicine. Additional complexity is introduced when refrigerated storage and blood component preservation are part of the experimental design. ${ }^{7}$ Refrigerated storage of RBC induces a series of biochemical and morphological modifications, collectively denoted "the storage lesion." ${ }^{8}$ For example, stored RBC progressively lose their capacity to cope with oxidant stress, ${ }^{9}$ which is paralleled by their decreased ability to sustain energy metabolism.

Metabolic investigations of human RBC units stored using all currently licensed storage additives ${ }^{10-12}$ helped to identify the impact of processing strategies (including leukoreduction ${ }^{13}$ and storage solutions ${ }^{14}$ ) on the molecular heterogeneity of stored units. Several factors complicate the study of the potential impact of "age of blood" on clinical outcomes. ${ }^{8}$ For example, units from some donors may store better than others for genetic, dietary, and/or environmental reasons, and the metabolic age of a RBC unit may differ from its chronological age. ${ }^{15}$ Findings from the Recipient Epidemiology and Donor evaluation Study (REDS-III) demonstrate donor-dependent heterogeneity in the propensity of $\mathrm{RBC}$ to hemolyze in vitro in response to storage duration, oxidative stress, and mechanical/osmotic insults. ${ }^{16}$ Therefore, improving RBC storage quality through increased understanding of $\mathrm{RBC}$ metabolism could enhance RBC quality for all donated units, independently of donor-specific factors, and improve transfusion outcomes overall.

Differences in storage quality become even more complex when comparing various animal species and strains; therefore, approximating human RBC function and storage outcomes is critical to pre-clinical, proof-of-concept studies. As such, it is important to use pre-clinical models of novel blood transfusion strategies that reliably approximate clinically relevant scenarios in humans. Although murine and canine models of blood storage and transfusion are available, ${ }^{17-21}$ macaques are generally perceived as a more relevant pre-clinical model, ${ }^{4}$ because of their evolutionary similarity to humans. For example, hematologic parameters in humans and RM are comparable. The RM hematocrit is $43 \pm 2 \%$ in males and $41 \pm 2 \%$ in females with corresponding hemoglobin levels of $13.1 \pm 0.9$ and $12.5 \pm 0.2 \mathrm{~g} / \mathrm{dL}$, respectively. ${ }^{22} \mathrm{RM}$ RBC distribution width and disc diameter are $13.0 \pm 0.7 \%$ and $8 \mu \mathrm{m}$, respectively, values similar to those of human $\mathrm{RBC} .^{22}$ Furthermore, the average life span of RM RBC is $98 \pm 21$ days, ${ }^{23,24}$ which is similar to that of human RBC (100-120 days), and significantly longer than that of murine RBC (55-60 days ${ }^{25}$.
However, little is known about refrigerated storage of RM $\mathrm{RBC}$ under standard blood banking conditions. Although prior preliminary studies explored similarities and differences in the proteomes of fresh RBC from mice, humans, and macaques, ${ }^{26}$ to the best of our knowledge, no previous study compared the metabolome and lipidome of human and RM RBC throughout 42 days of refrigerated storage. Thus, the current data provide a comparative analysis of RBC metabolic pathways and lipidomic changes occurring over time and identify dietary and environmental compounds unique to each species.

\section{Methods}

Extensive methodological details are provided in the Online Supplementary Methods.

\section{Blood collection, processing and storage}

Blood from 5-year old RM ( $\mathrm{n}=20 ; 10$ male/10 female) was collected into a syringe, using a $20 \mathrm{G}$ needle, from the femoral vein under ketamine/dexmedotomidine $(7 \mathrm{mg} / \mathrm{kg} / 0.2 \mathrm{mg} / \mathrm{kg}$ ) anesthesia according to the Food and Drug Administration (FDA) White Oak Animal Care and Use protocol 2018-31. All blood donor animals originated from the same colony located on Morgan Island, South Carolina and were naïve to experimentation at the time of blood collection.

The blood from 30- to 75-year old human volunteers ( $n=21$; 11 male/10 female) was collected into a syringe, using a $16 \mathrm{G}$ needle, from the median cubital vein under informed consent according to National Institutes of Health (NIH) study Institutional Research Board \#99-CC-0168 "Collection and Distribution of Blood Components from Healthy Donors for In Vitro Research Use" under an NIH-FDA material transfer agreement. Blood was collected into acid citrate dextrose, leukofiltered, and stored in AS-3 in pediatric-sized bags, designed to hold $20 \mathrm{~mL}$ volumes, which mimicked the composition of standard full-sized units (i.e., incorporating polyvinylchloride and phthalate plasticizers)

The $\mathrm{RBC}$ were stored at $4-6^{\circ} \mathrm{C}$ for 42 days. The $\mathrm{RBC}$ and supernatants were separated via centrifugation upon sterile sampling of each unit on days $0,7,14,21,28,35$, and 42 .

\section{Ultra-high pressure liquid chromatography - mass spectrometry metabolomics and lipidomics}

Frozen RBC aliquots of $50 \mu \mathrm{L}$ volume were extracted 1:10 in ice-cold extraction solution (methanol:acetonitrile:water 5:3:2). ${ }^{27}$ Samples were vortexed and insoluble material pelleted, as described elsewhere. ${ }^{28}$ Analyses were performed using a Vanquish UHPLC coupled online to a $Q$ Exactive mass spectrometer (Thermo Fisher, Bremen, Germany). Samples were analyzed using a $3 \mathrm{~min}$ isocratic condition ${ }^{29}$ or a 5,9 , and $17 \mathrm{~min}$ gradient, as described previously. ${ }^{30,31}$ Additional analyses, including untargeted analyses and fragment ion search (FISh) score calculation via mass spectrometry, ${ }^{2}$ were performed with Compound Discoverer 2.0 and LipidSearch (Thermo Fisher, Bremen, Germany). For targeted quantitative experiments, extraction solutions were supplemented with stable isotopelabeled standards, and endogenous metabolite concentrations were quantified against the areas calculated for heavy isotopologues for each internal standard. ${ }^{30,31}$ Graphs and statistical analyses (either a $t$-test or repeated measures analysis of variance) were prepared with GraphPad Prism 5.0 (GraphPad Software, Inc, La Jolla, CA, USA), GENE E (Broad Institute, Cambridge, MA, USA), and MetaboAnalyst 4.0..$^{32}$ 


\section{Results}

\section{Fresh red blood cells from macaques and humans differ metabolically}

Metabolomic analyses were performed on leukocytefiltered, fresh (day 0) RBC from healthy human volunteers $(\mathrm{n}=21)$ and RM $(\mathrm{n}=20)$ (Figure 1A; Online Supplementary Table S1). Significant differences between species were determined by partial least squares-discriminant analysis, $t$-test-informed hierarchical clustering, and volcano plots (Figure 1B-D, respectively). Significant changes were noted in the levels of purines (e.g., hypoxanthine, urate), arginine and sulfur metabolites (e.g., glutathionylcysteine, glutathione, phytochelatins), carnitines, and xenometabolites (e.g., caffeine) in fresh RBC from the two species (Figure 1D). In the light of these changes, correlation analyses (Spearman) were performed across all metabolites to define the level of metabolic linkage in RBC from either species (Figure 1E). Identifying correlates in one species (e.g., macaques), disruptions of such correlations, and generation of novel correlations indicate metabolic rewiring (Figure 1F, from left to right). These measurements can then be used to subtract the correlations observed for every pair of metabolites in each species, resulting in a differential heat map (Figure 1F, rightmost panel). This map highlights pathways that are preserved (Figure 1G, leftmost panel), and those that undergo metabolic rewiring in RBC from these species ( $>30 \% \Delta \mathrm{r}$ between species, $P<0.05$ ), such as those involving glutathione homeostasis, sulfur, purine, carboxylic acid, and arginine metabolism (Figure 1G).

\section{Metabolic tracing experiments with $\left[1,2,3-{ }^{13} C_{3}\right]$ glucose in fresh human or macaque red blood cells}

In light of metabolic differences in glutathione homeostasis observed at steady state, we hypothesized that species-specific alterations of glucose fluxes affect the NADPH-generating pentose phosphate pathway (PPP). $\mathrm{NADPH}$ is required to preserve RBC redox homeostasis by favoring reduction of glutathione and other reversibly oxidized thiols. To test this hypothesis, leukoreduced $\mathrm{RBC}$ lysates from RM $(\mathrm{n}=20)$ and humans $(\mathrm{n}=21)$ were incubated with $\left[1,2,3{ }^{13} \mathrm{C}_{3}\right]$ glucose for $1 \mathrm{~h}$ at $37^{\circ} \mathrm{C}$ (Figure 2 ). This allows comparisons of ${ }^{13} \mathrm{C}$ incorporation through glycolysis (+3 isotopologues) and the PPP (+2) in RM and human RBC at steady state (Figure 2; Online Supplementary Figure S1), as described previously.,93 Importantly, RM and human RBC showed similar rates of glucose consumption, along with comparable levels of the glycolytic products pyruvate and lactate, in the absence of any apparent preference in glycolysis/PPP fluxes (Online Supplementary Figure S1). However, early steps of glycolysis showed significantly different rates between these species, with higher levels of ${ }^{13} \mathrm{C}_{3}$-glucose 6-phosphate (and hexose phosphate isobars) in human RBC and higher levels of ${ }^{13} \mathrm{C}_{3}$-fructose bisphophate in RM (Figure 2). RM RBC showed significantly higher levels of ${ }^{13} \mathrm{C}$ accumulation in intermediates of the glyoxylate pathway (e.g., ${ }^{13} \mathrm{C}_{3}$-methylglyoxal and ${ }^{13} \mathrm{C}_{3^{-}}$and ${ }^{13} \mathrm{C}_{5}$-lactoyl-glutathione) and late PPP products (e.g., ${ }^{13} \mathrm{C}_{2}$-ribose), but lower levels of labeled glutathione and purines (e.g., ${ }^{13} \mathrm{C}_{2}-$ AMP) (Figure 2). Further focusing on reducing equivalents, ${ }^{34}$ there were species-specific preferences in substrates used to generate carboxylic acids, with ${ }^{13} \mathrm{C}_{3}$-malate preferred in $\mathrm{RM}$ and ${ }^{13} \mathrm{C}_{2}$-malate in humans (Figure 2).
Interspecies comparison of the red blood cell metabolome during refrigerated storage

Metabolomic analyses were performed on 574 samples of stored RBC and supernatants from RM and humans (Figure 3A; Online Supplementary Table S1). Partial least squares-discriminant analysis of RBC data showed significant species- and time-dependent clustering of these samples across principal component 1 (28.8\% of total variance) and principal component 2 (18.6\%), respectively (Figure $3 \mathrm{~B})$. The Venn diagram in Figure 3C, D shows the number of significant metabolites by repeated measures two-way analysis of variance and related pathway analyses. Hierarchical clustering analyses further highlighted significant storage- and species-dependent differences (Figure 3E; a vectorial version of this figure, including metabolite names, is provided in Online Supplementary Figure S2).

Targeted metabolomic analyses were accompanied by untargeted metabolomic analyses (Online Supplementary Figure $S 3 A-D$ ). Interestingly, these analyses expanded on the targeted metabolomic data by highlighting species-specific changes in levels of xenometabolites stored RBC (Online Supplementary Figure S3E) derived from personal habits (e.g., cotinine from smoking in 2 of 21 donors), chemical exposure (e.g., aniline, nitrosopiperidine), therapeutic drugs (e.g., acetaminophen, in 2 subjects), and diet (e.g., caffeine and theophylline in humans; methyl-histidine, phloionic acid, lupinine, gallocatechin, azelaic acid, and asarone in RM).

Although limited by the relatively small numbers of male and female human and RM donors evaluated, a preliminary breakdown by sex identified a significant impact in RM, especially regarding carboxylic acid, arginine, fatty acid, and purine metabolism (Online Supplementary Figure $S 4 A-D)$.

\section{Species-specific differences in metabolic phenotypes of stored red blood cells}

Significant differences were observed in RBC levels of sulfur-containing metabolites involved in one-carbon and glutathione metabolism (Figure 4), including taurine, Sadenosylmethionine (SAM), cysteine, cystathionine, and glutathione [both reduced (GSH) and oxidized (GSSG)]; all were higher in RM than human RBC throughout storage, except GSH. Increased glutathione pools and activation of the gamma-glutamylcycle, ascorbate metabolism, and glutaminolysis were observed in RM, as compared to human, RBC (Figure 4). These observations were not accompanied by significantly different levels of PPP intermediates (except for higher levels of the non-oxidative phase PPP metabolite sedoheptulose phosphate in humans). However, in contrast to what was observed in fresh RBC, stored RM RBC showed higher levels of intracellular glucose in supernatants and cells (Figure 4), despite comparable levels of intracellular and supernatant levels of lactate (i.e., $10 \%$ increase in RM, $P<0.05$ ) (Online Supplementary Table S1). Of note, human RBC showed lower levels of 2,3diphosphoglycerate, but higher levels of ATP, during the first 2 weeks of storage (Figure 4).

Expanding on these observations, a deeper focus on purine metabolism revealed significantly higher levels of all purines and purine-containing metabolites in RM RBC. However, dramatic species-specific changes in purine oxidation metabolites were observed (Figure 5A), with RM RBC showing significantly higher levels of hypoxanthine (accumulating during storage in both species) and human 
RBC showing significantly higher levels of urate and hydroxyisourate (decreasing during storage).

Purine oxidation and salvage of deaminated purines involve using the amine group from aspartate through a reaction that generates fumarate, a minimally active pathway in mature RBC. ${ }^{35}$ Consistent with dysregulation of purine oxidation and salvage, species-specific changes in the levels of aspartate (higher in RM) and fumarate (higher in humans) were noted, suggesting a decreased rate of salvage reactions in RM RBC (Figure 5B). Similarly, RBC lev- els of some carboxylic acids (e.g., malate, 2-hydroxyglutarate) were higher in humans, whereas others (e.g., citrate, $\alpha$-ketoglutarate, succinate, oxaloacetate, itaconate) were higher in RM (Figure 5B), suggesting species-specific differences in transamination reactions (e.g., those dependent on the activity of alanine and aspartate aminotransferases) or carboxylic acid metabolism via cytosolic isoforms of Krebs cycle enzymes, all of which are active in mature RBC. . $4,36-38^{-3}$

In cells with mitochondria, carboxylate metabolism is
A

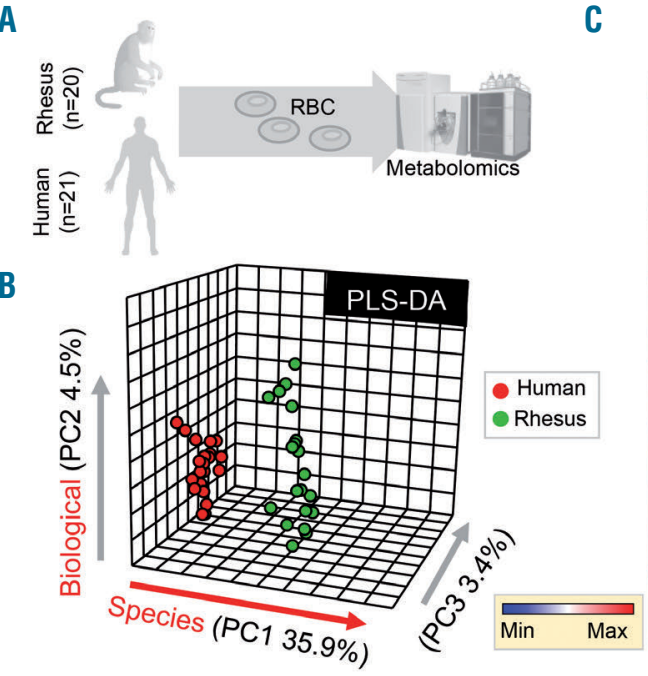

C

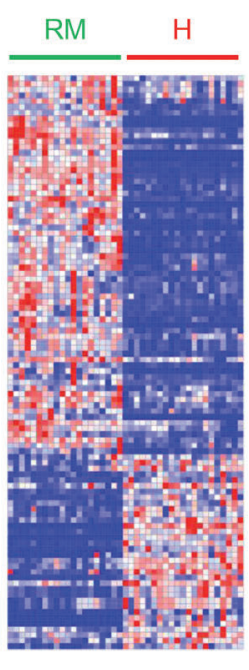

D

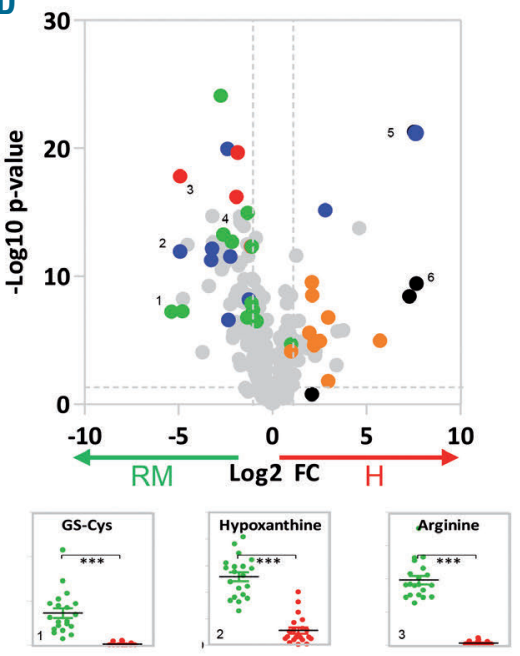

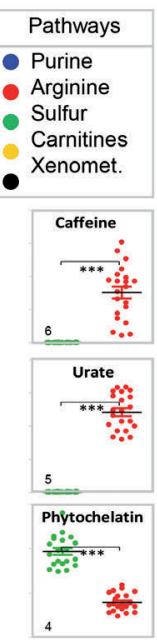

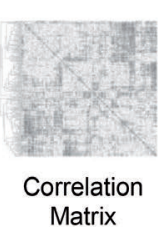

Matrix
Glutathione

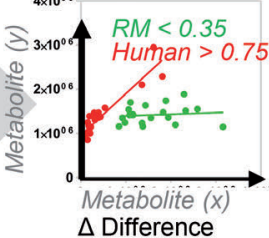
$\Delta$ Difference

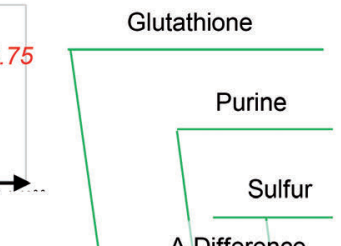

$\Delta$ Difference
F

Rhesus macaque HCA

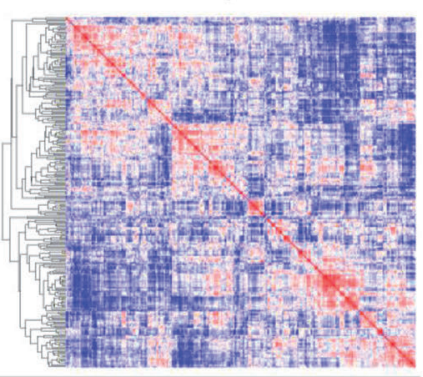

Human

(Same HCA order as RM)
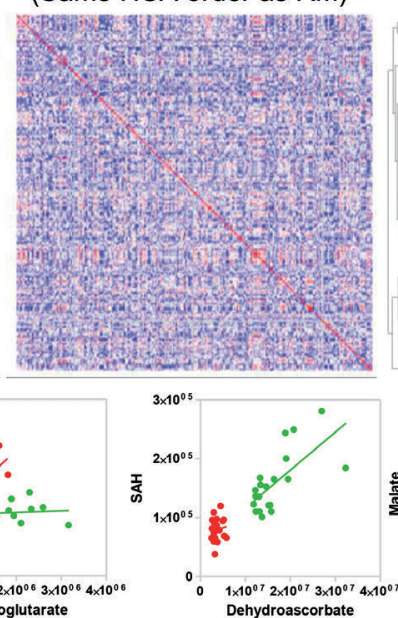

Dehydroascorbat

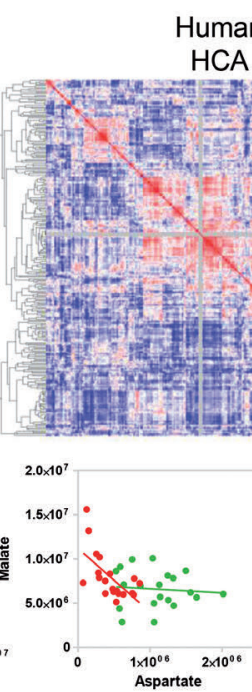

G

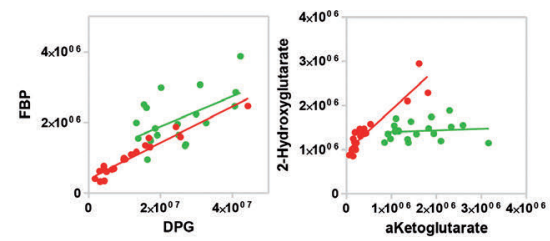

CA
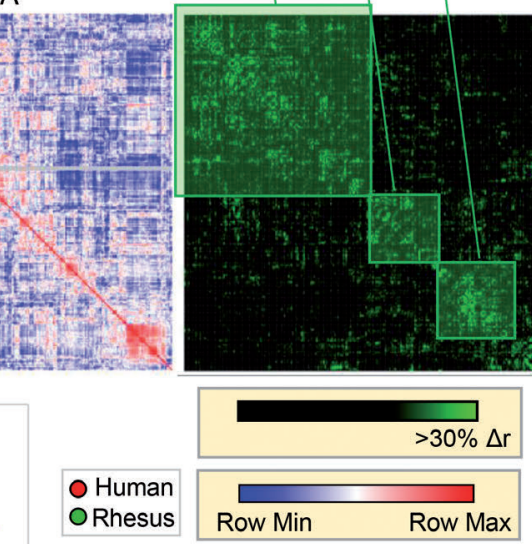

Figure 1. Metabolomics of fresh red blood cells from healthy human volunteers and Rhesus macaques. (A) An overview of the experimental design. (B) Partial least square-discriminant analysis (PLS-DA) of red blood cells from 21 humans (red) or 20 Rhesus macaques (RM) (green). (C) An overview of the metabolites that were significantly different between the two groups (a vectorial version of the heat map is provided in Online Supplementary Figure S2). (D) A volcano plot highlighting the major pathways differing between humans (red) and RM (green). (E) Metabolite levels were correlated among each other (Spearman) in humans (red) and RM (green). (F) These correlations were used to map hierarchical clustering profiles in RM and humans. RM and human RBC showed significantly different metabolic correlation maps. Metabolites whose linear correlations differed significantly between species ( $>30 \% \Delta r$ between species, $P<0.05)$ are highlighted in the rightmost panel in (F). (G) Some representative examples of correlations that are preserved (left) or lost (other panels) between species. PC: principal component; RBC: red blood cells; DPG: 2,3-diphosphoglycerate; HCA: hierarchical clustering analysis. 

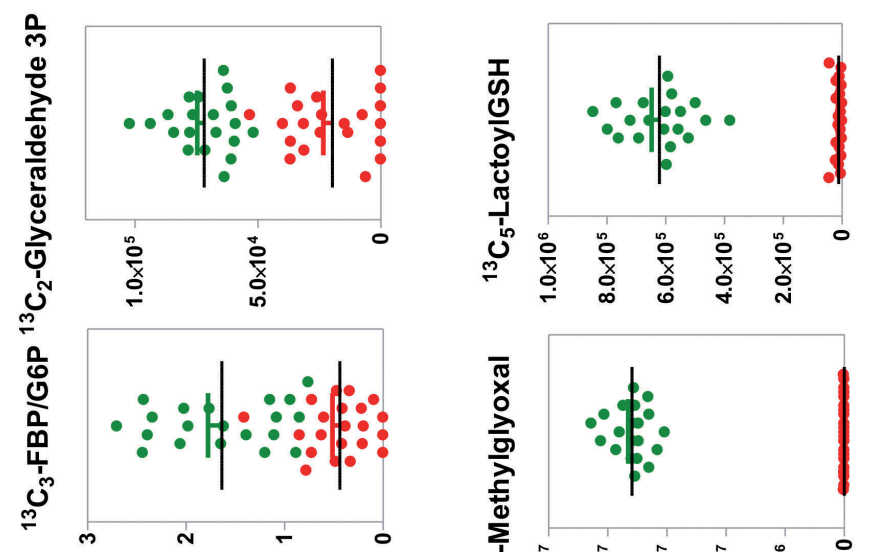

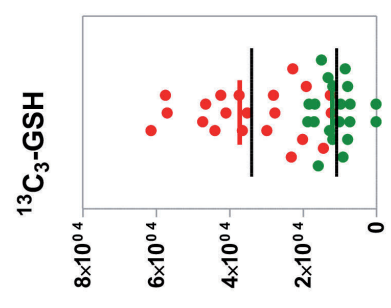
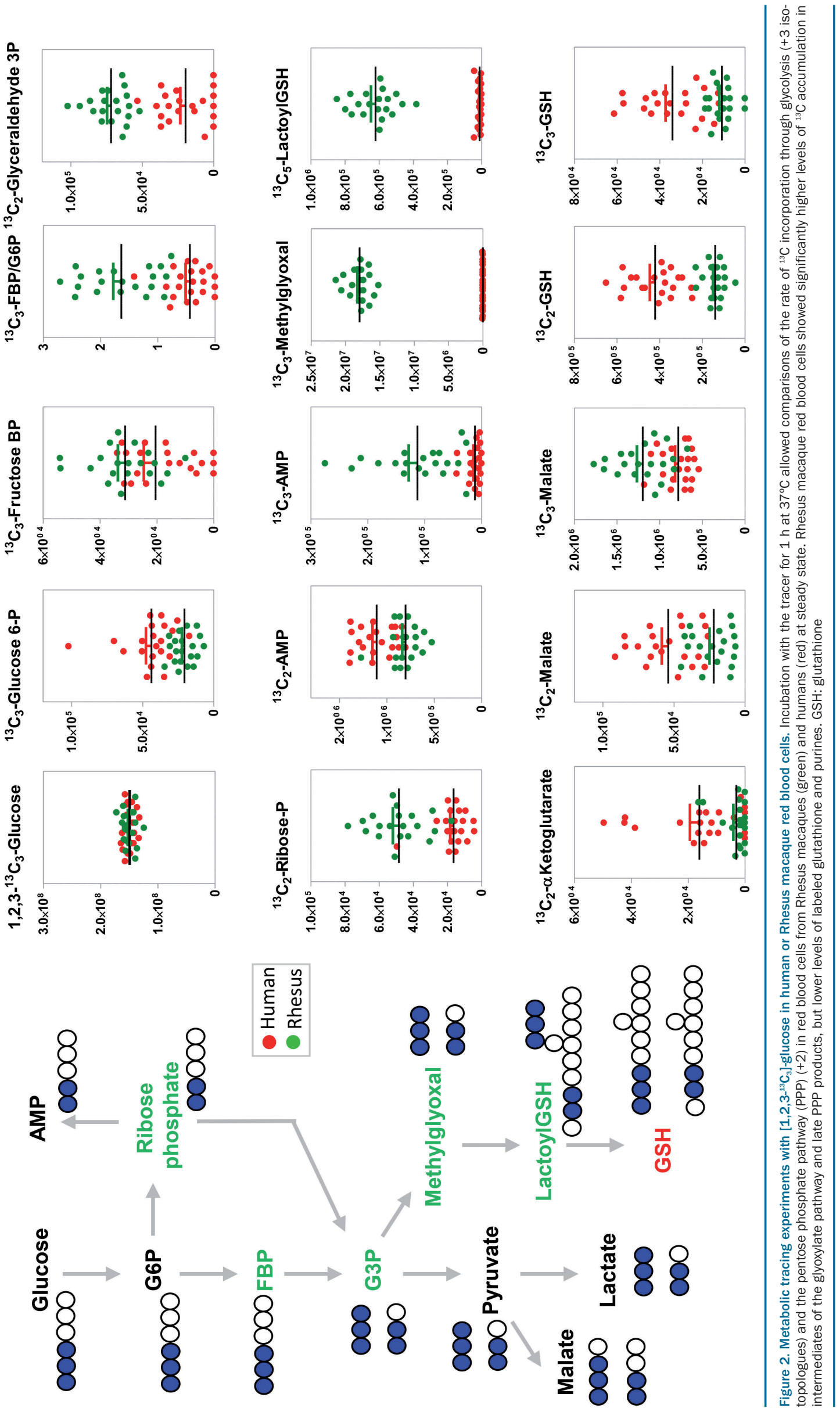
intertwined with the urea cycle, which is incomplete in mitochondria-devoid mature RBC. Arginine metabolism differed significantly between human and RM RBC, the latter having significantly higher RBC (and supernatant) levels of arginine, but with significantly lower levels of citrulline, ornithine, and creatine (Figure 5B). Although RM RBC had higher levels of polyamines (including sper- midine), these increases $(10-20 \%$ by storage day 42 ; $P<0.0025)$ were not sufficient to explain the $>100$-fold increase in arginine levels in RM RBC. However, comparable fold-change increases in asymmetric dimethylarginine (and isobaric isomers) were noted in RM RBC, as seen by untargeted metabolomics (Online Supplementary Figure S3C).

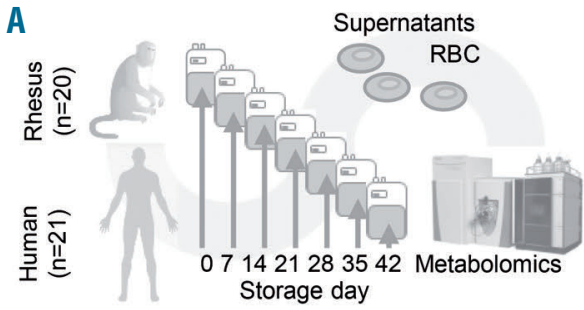

B

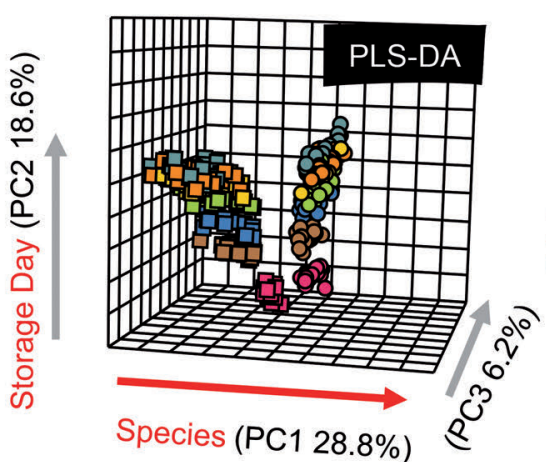

C

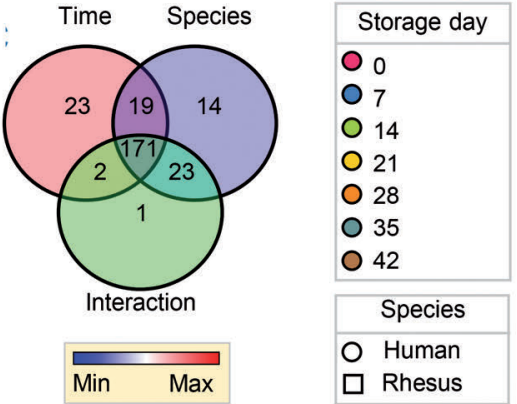

D

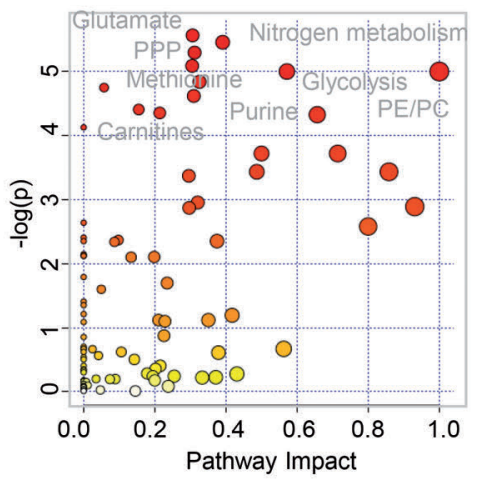

$\mathbf{E}$

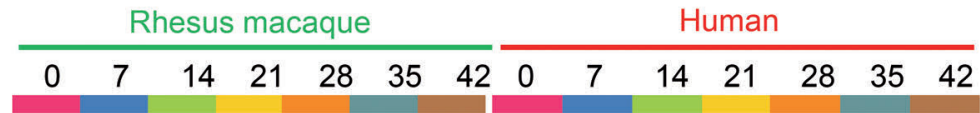

品
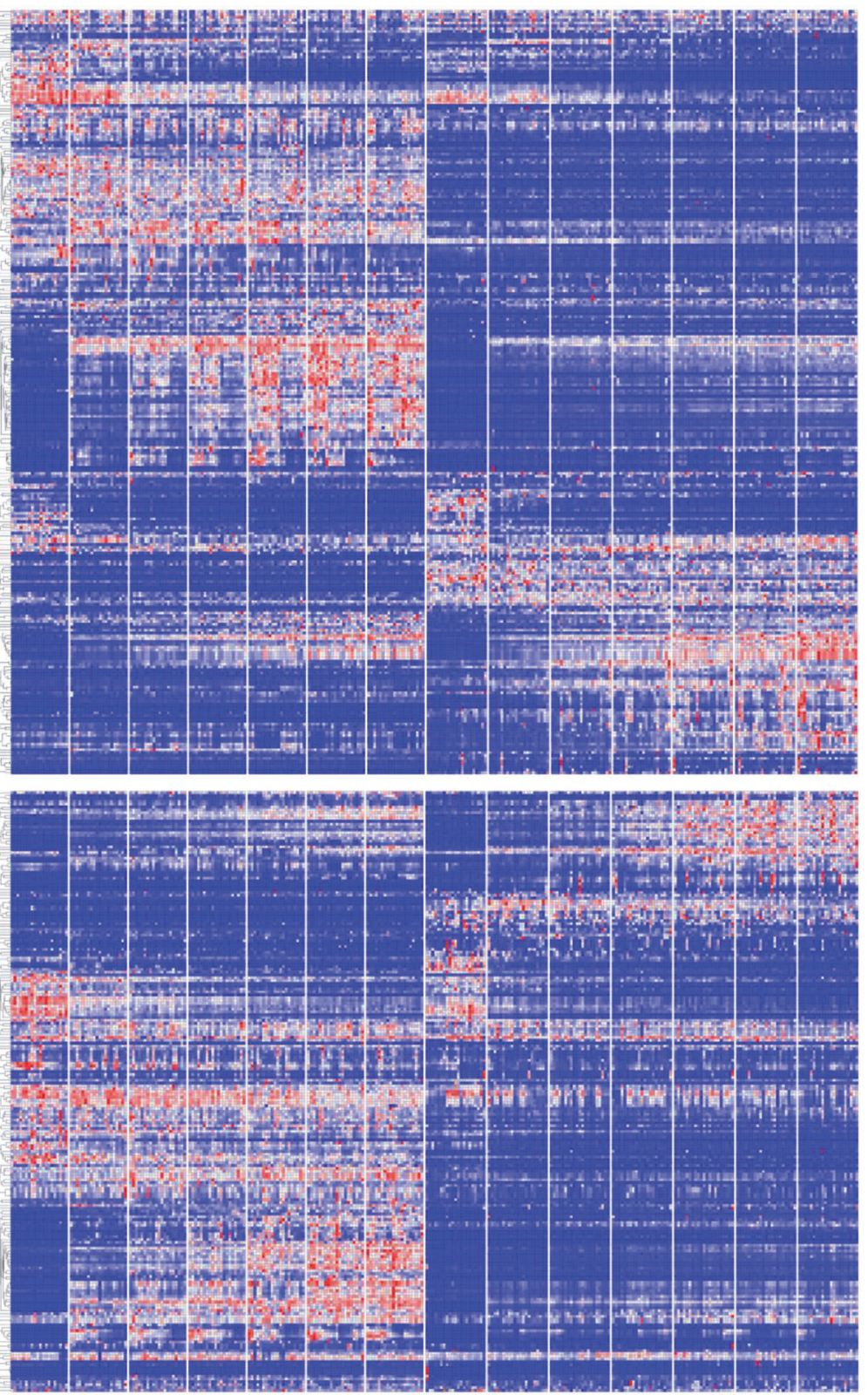

Figure 3. Interspecies comparison of the red blood cell metabolome during refrigerated storage. (A) Comparison of the metabolome of red blood cells (RBC) from humans and Rhesus macaques (RM). (B) Partial least squares-discriminant analysis (PLS-DA) shows significant species- and time-dependent clustering of samples across principal components (PC1 and PC2, respectively). (C, D) Venn diagram (C) showing the number of significant metabolites by repeated measures two-way analysis of variance and related pathway analyses (D). (E) A heat map showing significant metabolic changes in stored RBC and supernatants as a function of storage duration in humans and RM. A vectorial version of this figure is provided in Online Supplementary Figure S2. 
Storage-induced lipid remodeling and oxidant damage of membrane lipids is higher in Rhesus macaque, as compared to human, red blood cells

Interestingly, untargeted analyses revealed significantly higher levels of diethylhexyl-, monoethylhexyl- and free phthalate plasticizers in RM RBC as a function of storage (Online Supplementary Figure S3). Since both human and RM RBC samples were stored in the same polyvinylchloride units under identical conditions, we hypothesize that these results could, at least in part, be explained by differential species-specific storage-dependent membrane dynamics. Further analyses of targeted metabolomic data indicated that $\mathrm{RBC}$ acylcarnitines increased with storage duration. RM RBC were characterized by higher levels of short and medium chain acylcarnitines (C2-12), and lower levels of long and very-long acylcarnitines (C16-22 or longer), as compared to human RBC (Figure 6). Similarly, free fatty acids (medium and long-chain, but not very long-chain fatty acids) were higher in RM RBC (Figure 6), along with higher levels of sphingosine 1-phosphate and lipid peroxidation products, including 4hydroxynonenal (4-HNE) and its glutathionylated form (GS-HNE). Consistent with increased oxidant stress in stored RM RBC, along with tracing experiments in fresh RBC (Figure 2), RM RBC showed significantly higher storage-dependent increases in lactoyl-glutathione levels (Figure 6).

To expand on these observations, untargeted lipidomic analyses were performed on fresh (day 0 ) and end of storage (day 42) RM and human RBC (summarized by lipid classes in Online Supplementary Figure $S 5$ and volcano plots in Online Supplementary Figure S6A, B). Notably,

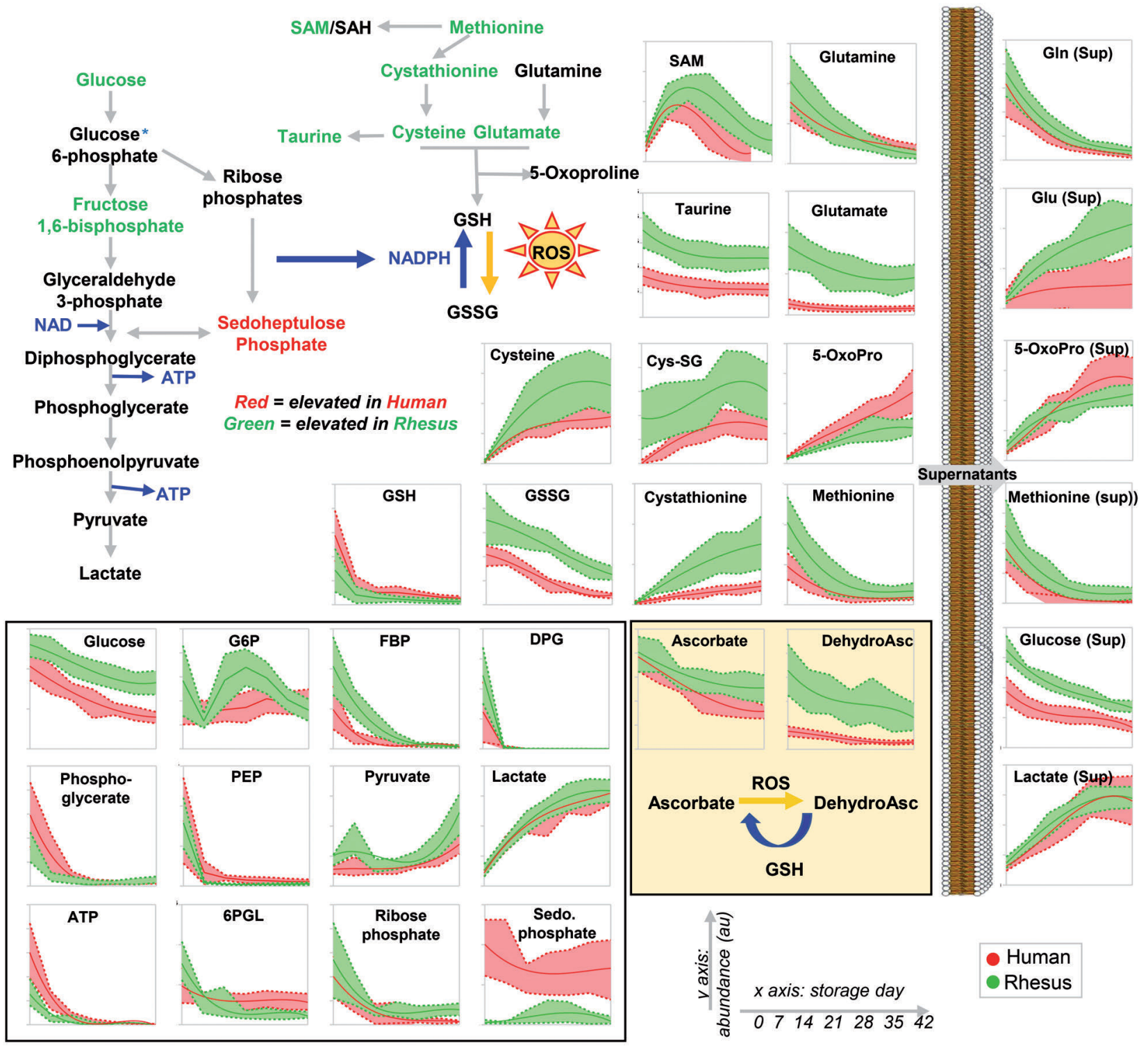

Figure 4. Species- and storage time-specific metabolic changes in Rhesus macaque and human red blood cells: focus on glycolysis, the pentose-phosphate pathway, glutathione, and one-carbon homeostasis. Data for Rhesus macaques are shown in green, those for humans are represented in red. Supernatant metabolites are shown in the right-hand side of the figure, outside the representative lipid bilayer of the cellular membrane. 
fresh and stored RM RBC had significantly higher levels of most phosphatidylserines (Online Supplementary Figure S6C-E) and short/medium-chain, but not long and verylong chain, fatty acyl-phosphatidylethanolamines (Online Supplementary Figure S6F, G).

\section{Validation using targeted quantitative metabolomics} and lipidomics

The initial, global approach generated extensive, but relative, quantification data for several pathways. To confirm critical observations, we performed validation exper- iments on all RBC and supernatant samples using targeted quantitative methods with stable isotope-labeled internal standards (Figure 7A). Results are reported in tabular and vectorial formats (Online Supplementary Table S2 and Online Supplementary Figure S7). There was a substantial overlap $\left(\mathrm{r}^{2}>0.75\right)$ between relative and absolute values for critical metabolites in amino acid, redox (glutathione and purine oxidation), and fatty acid metabolic pathways, which demonstrated significant differences between the two species as a function of storage duration (Figure 7B-F) and sex (Online Supplementary Figure S4E-G).

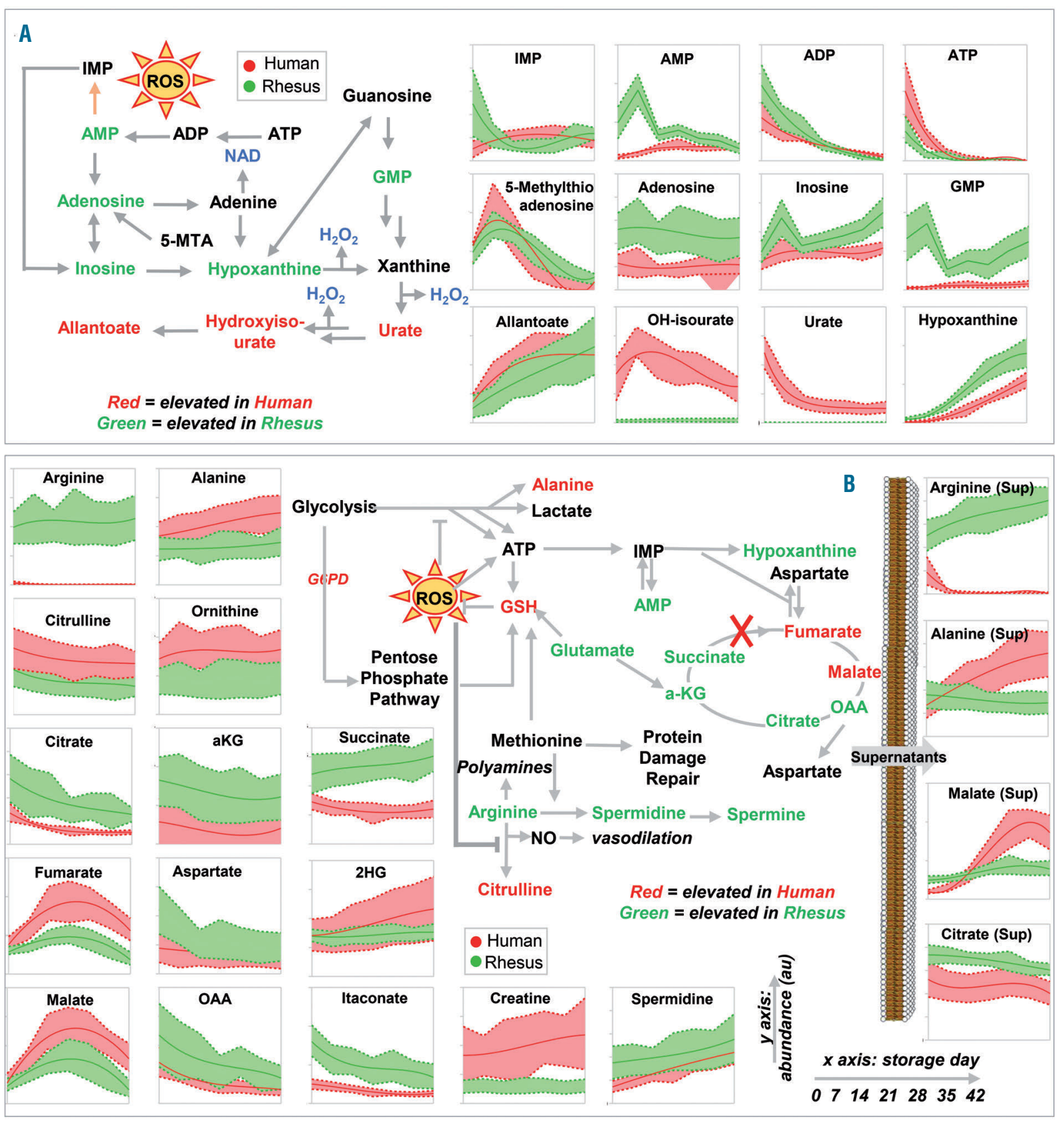

Figure 5. Species- and storage time-specific metabolic changes in Rhesus macaque and human red blood cells and supernatants: focus on purine metabolism, urea cycle, and carboxylate metabolism. (A, B) Data for Rhesus macaques are shown in green, those for humans are represented in red. Supernatant metabolites are shown in the right-hand side of panel $(B)$, outside the representative lipid bilayer of the cellular membrane. 


\section{Discussion}

Over the past two decades, animal models have helped to identify potential mechanisms critical to RBC storage biology and transfusion outcomes. Murine and canine models of RBC storage and transfusion were critical in identifying (i) etiological contributions to transfusionrelated acute lung injury, including the two-hit model; $;^{39}$ (ii) the impact of storage duration on mortality, ${ }^{21}$ and (iii) the role that iron overload plays in increasing the risk of septic complications. ${ }^{19}$ These models have limited genetic variability and a more homogeneous exposome than humans. This controlled strain-specific heterogeneity of genetic background in rodent models enables mechanistic studies to identify genetic and metabolic contributors to RBC storage quality and the post-transfusion performance of the RBC. ${ }^{17,18}$ In addition, animal models can be modified in a controlled fashion to allow for selective

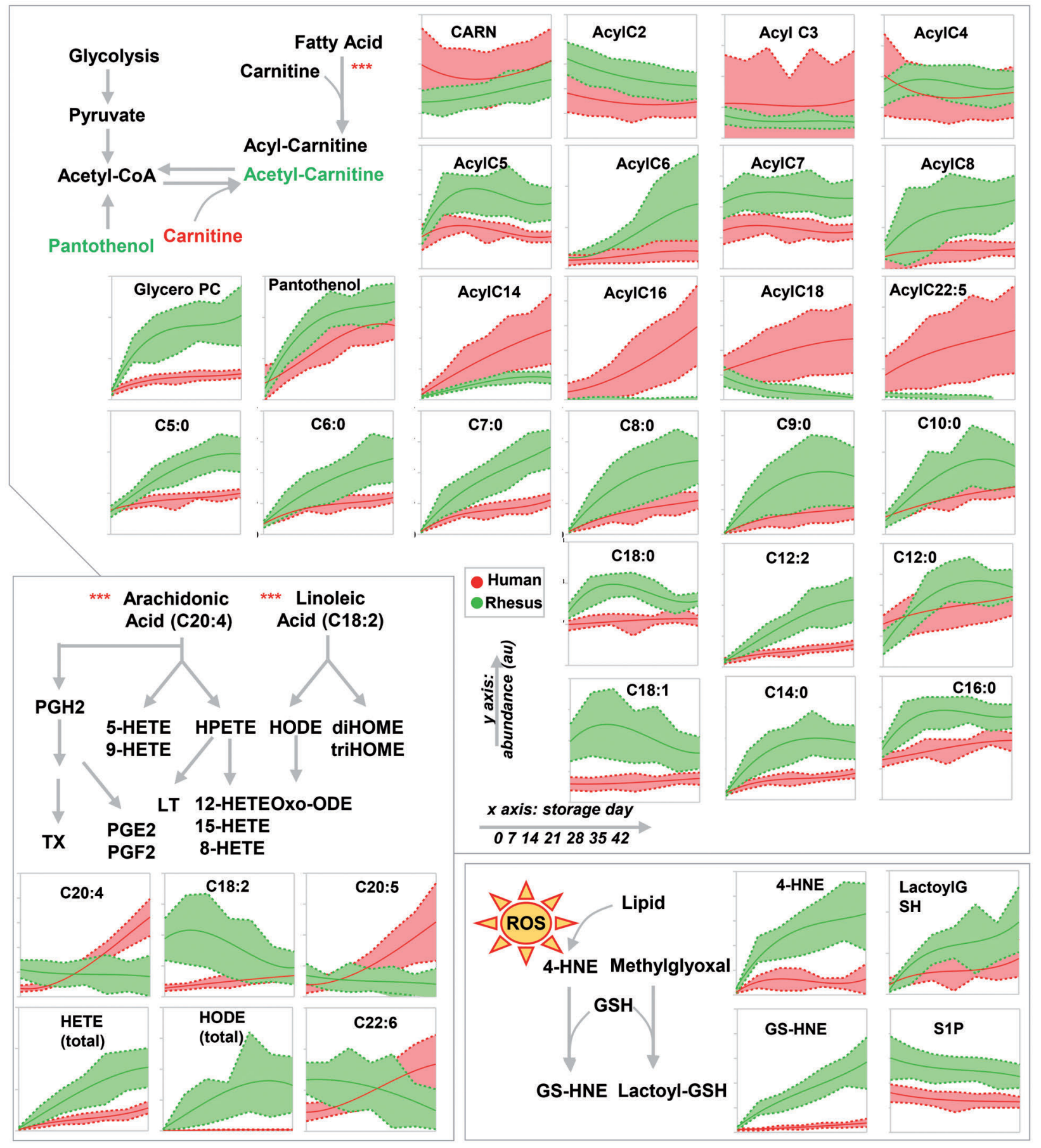

Figure 6. Species- and storage time-specific metabolic changes in Rhesus macaques and human red blood cells: focus on acyl-carnitines, free fatty acid metabolism, and lipid peroxidation products. Data for Rhesus macaques are shown in green, those for humans are represented in red. 
genetic, pharmacological, dietary, or surgical interventions, which are difficult, and sometimes impossible, to achieve with humans. Given their phylogenetic similarity to humans, RM may provide a particularly relevant $\mathrm{RBC}$ storage and transfusion model. For example, RM demon- strate similar pathobiology and metabolic derangements common to human trauma patients. ${ }^{2}$

By combining state-of-the-art targeted and untargeted metabolomics and lipidomics, as well as tracing experiments with $\left[1,2,3-{ }^{13} \mathrm{C}_{3}\right]$ glucose, the present study shows

A
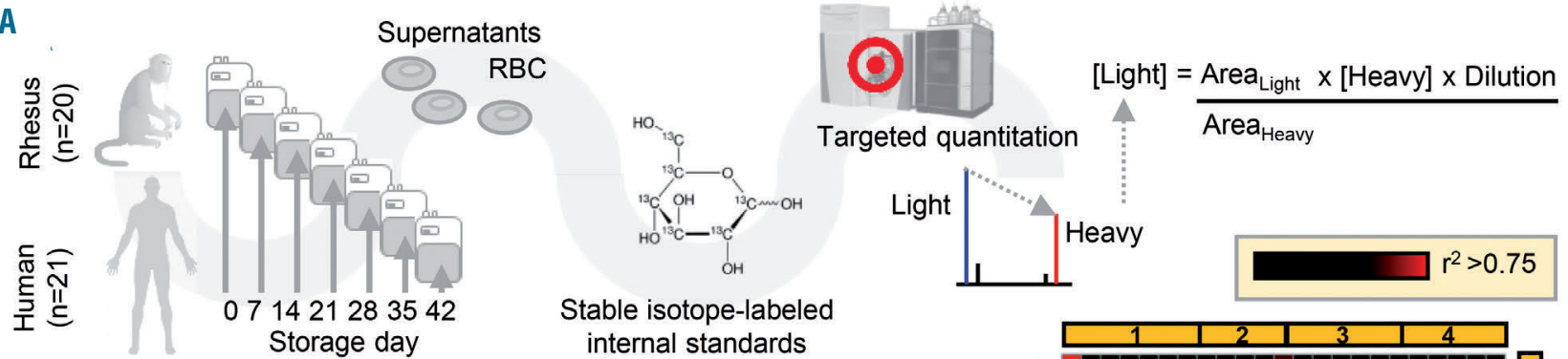

Stable isotope-labeled internal standards

B

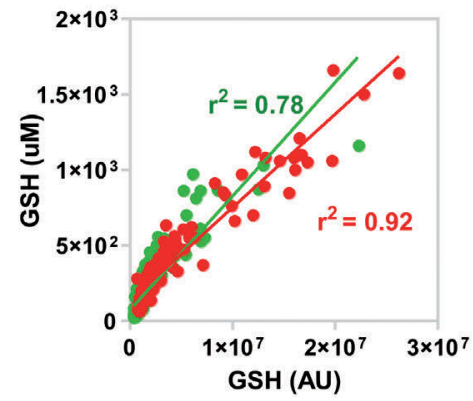

C

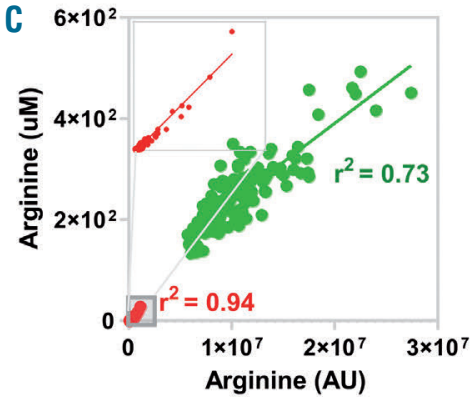
(1) Amino acids
(2) Purines
(3) Glutathione
(4) Fatty acids

Targeted quantitation $A$ Area ${ }_{\text {Heavy }}$<smiles>[Mg]=C1CCCCC1</smiles>

$E$

Rhesus macaque
Human

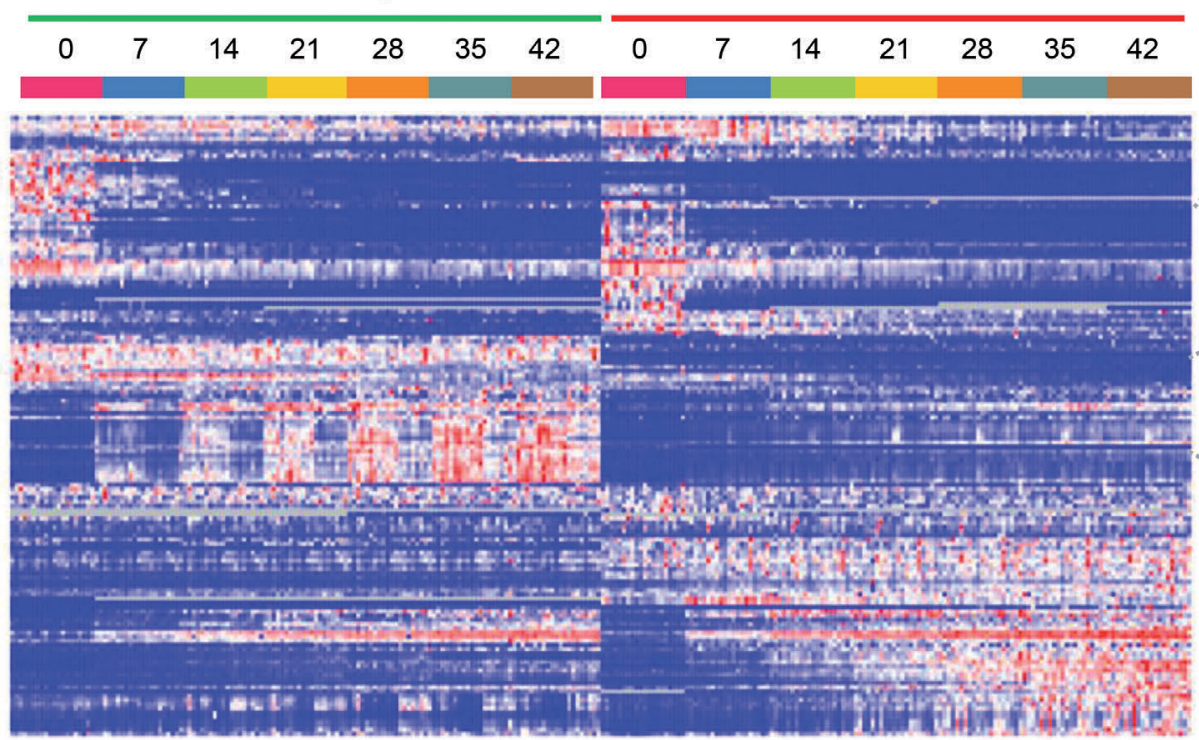

D

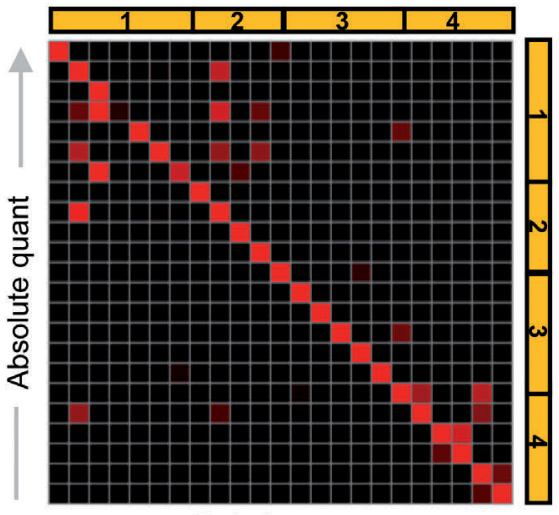

Relative quant
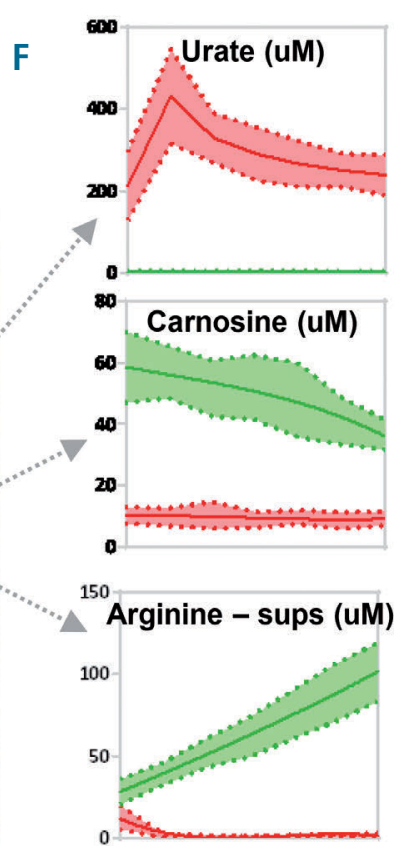

Figure 7. Validation experiments on red blood cells and supernatants. (A) Validation via targeted quantitative mass spectrometry-based measurements against stable isotope-labeled internal standards. (B, C) There was a substantial overlap of relative and absolute quantitative measurements for both metabolites showing similar interspecies concentrations (e.g., glutathione - GSH (B) and significantly different interspecies levels (e.g., arginine) (C). This is further exemplified in (D), in which red dots in the heat map show metabolites from different pathways (including amino acids, glutathione homeostasis, purines, and fatty acids), whose measurements by either method correlated significantly $\left(r^{2}>0.75\right)$. (E) Notably, significant metabolic changes during storage, illustrated in the heat map, recapitulate the relative quantification measurements in Figure 1 and related supplements. For example, quantitative measurements of urate, carnosine, and arginine are consistent with respective measurements from the exploratory analysis. All measurements are provided in tabular form in Online Supplementary Table S2 and in vectorial form in Online Supplementary Figure S7. 
that fresh and stored human and RM RBC have comparable glycolytic and PPP fluxes. However, RM RBC have higher baseline levels of glutathione oxidation and turnover (i.e., the gamma-glutamyl cycle), along with increased transamination reactions (e.g., higher levels of glutamine, aspartate, alanine, and glutamate) and sulfur metabolism (e.g., cysteine, taurine, and SAM). Glutathionylated lipid and sugar oxidation products (e.g., GS-HNE and lactoyl-glutathione) suggest that increased sulfur metabolism in this species could result from enhanced basal oxidant stress and/or decreased activity of the deglutathionylation protein machinery. These results were confirmed by tracing experiments, highlighting significant activation of the glyoxylate pathway in RM, as compared to human, RBC; this pathway is usually associated with oxidant stressors (e.g., diabetes ${ }^{40}$ ). Alternatively, this observation suggests that evolution has driven speciesspecific changes in sulfur metabolism resulting from sulfurrich diets in the wild, as suggested by increased plantderived ${ }^{41}$ phytochelatins in RM RBC. Combining targeted and untargeted metabolomics identified additional dietary metabolites of plant origin in RM, supporting a significant impact of diet on the molecular composition of their RBC. Based on this observation, one could test whether differences in free fatty acids, fatty acyl composition of carnitines and phosphatidylethanolamines (but not phosphatidylserines) in RM RBC result from differing fatty acyl contents of their dietary lipids, as compared to humans. Since fatty acyl-conjugation to carnitines depends on highenergy ATP and co-enzyme A availability, and is involved in lipid damage repair, one could speculate that differential levels of acyl-carnitines in humans and RM could be explained by a differential species-specific capacity to preserve ATP stores or prevent/repair lipid damage during storage. In this view, species-specific differences in carnitine metabolism suggest a differential impact of storage on membrane phospholipid homeostasis. ${ }^{42}$ Such phenomena may be explained, in part, by simply considering that the $\mathrm{RBC}$ storage additive used here was designed to optimize human RBC storage. Higher phosphatidylserine levels in RM RBC suggest the potential for increased erythrophagocytosis by mononuclear phagocytes; this could be tested by post-transfusion RBC recovery studies. Nonetheless, the present study assessed the total RBC phosphatidylserine content, rather than its cellular compartmentalization (i.e., exposure on the RBC membrane outer leaflet ${ }^{43}$ ). On the other hand, phthalate plasticizers, which accumulate up to millimolar levels in human RBC by the end of storage, appeared to be present at even higher levels in RM RBC, despite virtually identical storage conditions; one possible explanation, consistent with the current data, is an increase in lipid oxidation and remodeling in RM RBC.

Xenometabolites, including environmental and dietary metabolites, can affect RBC integrity. Interestingly, all human blood donors in this study consumed caffeine (a purine metabolite that could modulate stored RBC metabolism via signaling through adenosine receptors ${ }^{44}$ ), based on detecting the parent compound and its metabolites. Additionally, cotinine (a nicotine metabolite and marker of smoking ${ }^{45}$ ) was detected in two of 21 donors, similar to the overall smoking incidence in USA donors (14\% in 2017). Similarly, metabolites of chemical exposure, such as aniline, were only detected in human donor RBC, with broad inter-donor variability.

Sex-specific signatures were detected, especially in RM, consistent with recent observations about the potential impact of sex on RBC storability and capacity to cope with oxidant and osmotic stressors; these parameters appear to be improved in female donor RBC. . $^{16,40}$ Metabolic pathways affected by sex include arginine, carboxylic acid metabolism, and purine oxidation, which were validated using targeted quantitative measurements.

Perhaps the study's most interesting finding is that purine oxidation products were all higher in RM, as compared to human, RBC, except for the antioxidant urate. ${ }^{47}$ This is particularly relevant in light of the recently described role of ATP breakdown and oxidation products upstream of urate (e.g., hypoxanthine as a critical marker of post-transfusion recovery in humans ${ }^{35}$ ). Similarly, arginine and asymmetric dimethylarginine (isobaric isomers could not be resolved in this study) were $>100$-fold higher in RM RBC throughout storage. This suggests that RM RBC have an altered capacity for metabolizing arginine to ornithine or citrulline via arginase and nitric oxide synthase, a phenomenon previously connected to a potential impact of blood storage and transfusion on nitric oxide metabolism and transfusion-related vasodilatory capacity. ${ }^{48}$

There are limitations to the study despite our attempt to keep blood experimental conditions constant between RM and humans. For example, blood collection from RM required mild anesthesia/sedation with ketaminedexmedetomidine. In one study, anesthetics and sedatives alone or in combination transiently increased circulating glucose levels and promoted insulin resistance (within the hour after dosing). ${ }^{49}$ Although we are unaware of any association between donor age and RBC storage quality, the RM RBC evaluated in this study were donated by adolescent or young adult animals, whereas the human donors represented the general age range of individuals volunteering for blood donation. The RM RBC were collected from animals originating on Morgan Island, South Carolina, whose ancestors were transferred from the Caribbean Primate Research Center in Puerto Rico in 1979-1980. The Morgan Island RM form a large, free-ranging colony, with more than 4,000 animals and $\sim 75 \%$ female predominance. ${ }^{50}$ The colony comprises animals that descended from India with little or no known Chinese RM introgression. Although most large national primate research centers in the USA conduct extensive genetic testing of animals in their care to promote gene flow, diverse genetic composition remains a challenge. Conversely, little genetic testing was performed on the Morgan Island colony. Nonetheless, although it is not expected that mixed Chinese-India hybrid animals occur there, it is likely that this colony experiences some genetic drift and genetic homozygosity due to the potential for inbreeding.

\section{Conclusion}

By describing the metabolic landscape of human and RM RBC throughout 42 days of refrigerated storage, we identified storage-, diet-, and sex-specific metabolites that may affect human biology and the potential translatability of future pre-clinical studies using RM as a model for RBC storage and transfusion. Although species-specific differences were certainly anticipated, identifying molec- 
ular similarities and differences in metabolic phenotypes of fresh and stored RBC when comparing these two species will inform the appropriateness of RM as a model in transfusion medicine and, specifically, of RBC storage in the context of specific interventions (e.g., testing novel storage additives). As such, our results suggest that several metabolic pathways in RM RBC overlap those in human RBC at baseline and during storage. This assessment of comparative RBC biology will likely be relevant in pre-clinical and clinical transfusion medicine and hematology. Nonetheless, clear differences emerged in this initial comparison, which provide opportunities for further investigation of uniquely different biochemical pathways that affect RBC under baseline conditions and during refrigerated storage.

\section{Acknowledgments}

Research reported in this publication was supported by funds from the Boettcher Webb-Waring Investigator Award (to ADA), RM1GM131968 from the National Institute of General and Medical Sciences (to $A D A$ ), R01HL146442 (to $A D A$ ) and R01HL148151 (to SLS, ADA, and JCZ) from the National Heart, Lung and Blood Institutes (to $A D A$ ) and a Shared Instrument grant from the National Institute of Health (S10OD021641).

\section{References}

1. Rhesus Macaque Genome Sequencing and Analysis Consortium, Gibbs RA, Rogers J, et al. Evolutionary and biomedical insights from the rhesus macaque genome. Science. 2007;316(5822):222-234

2. Reisz JA, Wither MJ, Moore EE, et al. All animals are equal but some animals are more equal than others: plasma lactate and succinate in hemorrhagic shock - a comparison in rodents, swine, nonhuman primates, and injured patients. J Trauma Acute Care Surg. 2018;84(3):537-541.

3. Hatziioannou T, Ambrose Z, Chung NPY, et al. A macaque model of HIV-1 infection. Proc Natl Acad Sci U S A. 2009;106 (11):4425-4429.

4. Lankau EW, Turner PV, Mullan RJ, Galland GG. Use of nonhuman primates in research in North America. J Am Assoc Lab Anim Sci. 2014;53(3):278-282.

5. D'Alessandro A, Moore HB, Moore EE, et al. Early hemorrhage triggers metabolic responses that build up during prolonged shock. Am J Physiol Regul Integr Comp Physiol. 2015;308(12):R1034-1044.

6. Schwarz H, Dorner F. Karl Landsteiner and his major contributions to haematology. $\mathrm{Br} \mathrm{J}$ Haematol. 2003;121(4):556-565.

7. Waterman HR, Kapp LM, Howie HL, et al. Analysis of 24-h recovery of transfused stored RBCs in recipient mice of distinct genetic backgrounds. Vox Sang. 2015;109(2): 148-154.

8. Yoshida T, Prudent M, D'Alessandro A. Red blood cell storage lesion: causes and potential clinical consequences. Blood Transfus. 2019;17(1):27-52

9. Reisz JA, Wither MJ, Dzieciatkowska M, et al. Oxidative modifications of glyceraldehyde 3-phosphate dehydrogenase regulate metabolic reprogramming of stored red blood cells. Blood. 2016;128(12):e32-42.

10. D'Alessandro A, Reisz JA, Culp-Hill R, et al. Metabolic effect of alkaline additives and guanosine/gluconate in storage solutions for red blood cells. Transfusion. 2018;58(8): 1992-2002.

11. Rolfsson Ó, Sigurjonsson ÓE, Magnusdottir $\mathrm{M}$, et al. Metabolomics comparison of red cells stored in four additive solutions reveals differences in citrate anticoagulant permeability and metabolism. Vox Sang. 2017;112(4):326-335

12. Cancelas JA, Dumont LJ, Maes LA, et al. Additive solution-7 reduces the red blood cell cold storage lesion. Transfusion. 2015;55(3):491-498.

13. Pertinhez TA, Casali E, Baroni F, et al. A comparative study of the effect of leukoreduction and pre-storage leukodepletion on red blood cells during storage. Front $\mathrm{Mol}$ Biosci. 2016;3:13.

14. D'Alessandro A, Culp-Hill R, Reisz JA, et al. Heterogeneity of blood processing and storage additives in different centers impacts stored red blood cell metabolism as much as storage time: lessons from REDS III Omics. Transfusion. 2019;59(1):89-100.

15. D'Alessandro A, Zimring JC, Busch $M$ Chronological storage age and metabolic age of stored red blood cells: are they the same? Transfusion. 2019;59(5):1620-1623.

16. Kanias T, Lanteri MC, Page GP, et al. Ethnicity, sex, and age are determinants of red blood cell storage and stress hemolysis: results of the REDS-III RBC-Omics study. Blood Adv. 2017;1(15):1132-1141.

17. Zimring JC, Smith N, Stowell SR, et al. Strain-specific red blood cell storage, metabolism, and eicosanoid generation in a mouse model. Transfusion. 2014;54(1):137-148.

18. de Wolski $\mathrm{K}, \mathrm{Fu} \mathrm{X}$, Dumont LJ, et al Metabolic pathways that correlate with post-transfusion circulation of stored murine red blood cells. Haematologica. 2016;101(5): 578-586.

19. Hod EA, Zhang N, Sokol SA, et al. Transfusion of red blood cells after prolonged storage produces harmful effects that are mediated by iron and inflammation. Blood. 2010;115(21):4284-4292.

20. Klein HG. The red cell storage lesion(s): of dogs and men. Blood Transfus. 2017;15 (2):107-111

21. Solomon SB, Wang D, Sun J, et al. Mortality increases after massive exchange transfusion with older stored blood in canines with experimental pneumonia. Blood. 2013;121 (9):1663-1672.

22. Chen Y, Qin S, Ding Y, et al. Reference values of clinical chemistry and hematology parameters in rhesus monkeys (Macaca mulatta). Xenotransplantation. 2009;16(6): 496-501.

23. Fonseca LL, Alezi HS, Moreno A, et al. Quantifying the removal of red blood cells in Macaca mulatta during a Plasmodium coatneyi infection. Malar J. 2016;15(1):410.

24. Fonseca LL, Joyner CJ, Saney CL, et al. Analysis of erythrocyte dynamics in Rhesus macaque monkeys during infection with Plasmodium cynomolgi. Malar J. 2018; 17(1):410.

25. Kaestner L, Minetti G. The potential of erythrocytes as cellular aging models. Cell Death Differ. 2017;24(9):1475-1477

26. Pasini EM, Kirkegaard M, Mortensen $P$ Mann M, Thomas AW. Deep-coverage rhe- sus red blood cell proteome: a first comparison with the human and mouse red blood cell. Blood Transfus. 2010;8(Suppl 3):s126139.

27. Reisz JA, Nemkov T, Dzieciatkowska M, et al. Methylation of protein aspartates and deamidated asparagines as a function of blood bank storage and oxidative stress in human red blood cells. Transfusion. 2018;58(12):2978-2991.

28. Nemkov T, Hansen KC, Dumont LJ, D'Alessandro A. Metabolomics in transfusion medicine. Transfusion. 2016;56(4):980 993.

29. Nemkov T, Hansen KC, D'Alessandro A. A three-minute method for high-throughput quantitative metabolomics and quantitative tracing experiments of central carbon and nitrogen pathways. Rapid Commun Mass Spectrom Rapid Commun Mass Spectrom. 2017;31(8):663-673

30. Fu X, Felcyn JR, Odem-Davis K, Zimring JC Bioactive lipids accumulate in stored red blood cells despite leukoreduction: a targeted metabolomics study. Transfusion. 2016:56(10):2560-2570.

31. D'Alessandro A, Nemkov T, Yoshida T, et al Citrate metabolism in red blood cells stored in additive solution-3. Transfusion. 2017;57(2):325-336.

32. Chong J, Soufan O, Li C, et al MetaboAnalyst 4.0: towards more transparent and integrative metabolomics analysis Nucleic Acids Res. 2018;46(W1):W486 W494.

33. D'Alessandro A, Nemkov T, Yoshida T, et al Citrate metabolism in red blood cells stored in additive solution-3. Transfusion. 2017;57(2):325-336

34. Nemkov T, Sun K, Reisz JA, et al Metabolism of citrate and other carboxylic acids in erythrocytes as a function of oxygen saturation and refrigerated storage. Front Med. 2017;4:175

35. Nemkov T, Sun K, Reisz JA, et al. Hypoxia modulates the purine salvage pathway and decreases red blood cell and supernatant levels of hypoxanthine during refrigerated storage. Haematologica. 2018;103(2):361-372.

36. Nemkov T, Hansen KC, D'Alessandro A. A three-minute method for high-throughput quantitative metabolomics and quantitative tracing experiments of central carbon and nitrogen pathways. Rapid Commun Mass Spectrom Rapid Commun Mass Spectrom. 2017;31(8):663-673

37. D'Alessandro A, Dzieciatkowska M Nemkov T, Hansen KC. Red blood cell proteomics update: is there more to discover Blood Transfus. 2017;15(2):182-187. 
38. Reisz JA, Slaughter AL, Culp-Hill R, et al. Red blood cells in hemorrhagic shock: a critical role for glutaminolysis in fueling alanine transamination in rats. Blood Adv. 2017;1(17):1296-1305.

39. Silliman CC, Paterson AJ, Dickey WO, et al. The association of biologically active lipids with the development of transfusion-related acute lung injury: a retrospective study. Transfusion. 1997;37(7):719-726.

40. Knight J, Wood KD, Lange JN, Assimos DG, Holmes RP. Oxalate formation from glyoxal in erythrocytes. Urology. 2016;88:226.

41. D'Alessandro A, Taamalli M, Gevi F, et al. Cadmium stress responses in Brassica juncea: hints from proteomics and metabolomics. J Proteome Res. 2013;12 (11):4979-4997.

42. Tokarska-Schlattner M, Epand RF, Meiler F, et al. Phosphocreatine interacts with phospholipids, affects membrane properties and exerts membrane-protective effects. PloS One. 2012;7(8):e43178.

43. Nguyen DB, Wagner-Britz L, Maia S, et al. Regulation of phosphatidylserine exposure in red blood cells. Cell Physiol Biochem. 2011;28(5):847-856

44. Sun K, D'Alessandro A, Xia Y. Purinergic control of red blood cell metabolism: novel strategies to improve red cell storage quality. Blood Transfus. 2017;15(6):535-542.

45. Vuk T, O i T, Juki I. Influence of cigarette smoking on haemoglobin concentration - do we need a different approach to blood donor selection? Transfus Med. 2019;29(Suppl 1):70-71.

46. Kanias T, Sinchar D, Osei-Hwedieh D, et al. Testosterone-dependent sex differences in red blood cell hemolysis in storage, stress, and disease. Transfusion. 2016;56(10):2571 2583.

47. Tzounakas VL, Karadimas DG, Anastasiadi AT, et al. Donor-specific individuality of red blood cell performance during storage is partly a function of serum uric acid levels. Transfusion. 2018;58(1):34-40

48. D'Alessandro A, Reisz JA, Zhang Y, et al Effects of aged stored autologous red blood cells on human plasma metabolome. Blood Adv. 2019;3(6):884-896.

49. Monestier M, Bona CA. Antibodies possessing multiple antigen specificities and exhibiting extensive idiotypic cross-reactivity. Int Rev Immunol. 1988;3(1-2):59-70.

50. Taub DM, Mehlman PT. Development of the Morgan Island rhesus monkey colony. $\mathrm{P}$ R Health Sci J. 1989;8(1):159-169. 